\title{
¿No es país para viejos? La edad como criterio de triaje durante la pandemia COVID-19*
}

\author{
Jon Rueda Etxebarria \\ Universidad de Granada \\ ruetxe@ugr.es
}

Fecha de recepción: $15-6-2020$

Fecha de aceptación: 30-6-2020

\section{Resumen}

La pandemia de la COVID-19 ha levantado sospechas de edadismo y gerontofobia en diversas prácticas de racionamiento sanitario. La edad es un criterio de triaje controvertido. En este artículo se esclarece la relevancia ética de la edad dentro de los sistemas de triaje, analizando particularmente su rol dentro de los principios de equidad y de eficiencia. La equidad requiere dar más oportunidades a aquellos que han cumplido menos ciclos vitales. La eficiencia tiene en cuenta la edad de manera subrepticia al tratar de maximizar los beneficios a corto plazo (salvar vidas) y a largo plazo (salvar años de vida). Finalmente, la edad no debe ser la última ratio de las decisiones de triaje, aunque es una variable a tener en cuenta debido a su presencia implícita en principios fundamentales de priorización.

Palabras clave: bioética práctica; COVID-19; edadismo; fair innings; gerontofobia; priorización; racionamiento

Abstract. No country for old men?: Age as a triage criterion during the COVID-19 pandemic

The COVID-19 pandemic has raised suspicions of ageism and gerontophobia in various health rationing practices. Age is a controversial triage criterion. This article clarifies the ethical relevance of age within triage systems, particularly its role within the principles of fairness and efficiency. Fairness requires giving more opportunities to those who have completed fewer life cycles. Efficiency takes age into account surreptitiously when trying to maximize short-term (saving lives) and long-term (saving years of life) benefits. Finally, age should not be the final rationale in triage decisions, although it is a variable to be taken into account because of its implicit presence in fundamental principles of prioritization.

Keywords: ageism; COVID-19; fair innings; gerontophobia; practical bioethics; prioritization; rationing

* Agradezco los valiosos comentarios de María Teresa López de la Vieja, Belén Liedo y David Rodríguez-Arias, del colectivo ESPACyOS, y de los dos revisores anónimos de la revista ENRAHONAR. El proyecto que dio lugar a estos resultados recibió el apoyo de una beca de la Fundación "la Caixa" (ID 100010434). El código de la beca es LCF/BQ/DR20/11790005. También agradezco la financiación del proyecto BIOethAI+ (FFI2016-79000-P), financiado por el Ministerio de Economía, Industria y Competitividad del Gobierno de España. Este artículo expresa la visión propia del autor y en ningún caso representa la perspectiva de las entidades financiadoras. 


\section{Sumario}

1. Introducción: ¿Edadismo en la pandemia?

2. Clarificando el contexto de debate: de los criterios de triaje al triaje de criterios

3. Equidad, ciclos de vida y justicia intergeneracional
4. Eficiencia en la escasez: maximizando el beneficio de los recursos disponibles

5. Conclusión: la edad como categoría no excluyente

Referencias bibliográficas

\section{Introducción: ¿Edadismo en la pandemia?}

La pandemia de la COVID-19 despierta dos lecturas a primera vista disonantes sobre la protección dispensada a los miembros más veteranos de nuestras comunidades. Por un lado, el confinamiento obligatorio y la correspondiente paralización de la mayoría de las actividades laborales han supuesto un esfuerzo hercúleo. Esta es una respuesta social e institucional sin precedentes en lo que va de siglo, en la que se han sacrificado muchos intereses de primer orden y de gran valor agregado. Estas medidas de salud pública buscan mitigar el colapso sanitario y no poner en riesgo a grupos poblacionales vulnerables, entre los que las personas de edad provecta ocupan un lugar protagonista. Por otro lado, sin embargo, el uso de la edad como criterio excluyente en algunos dilemas de triaje ha despertado un gran eco mediático y sospechas de edadismo - discriminación injustificada por edad-. Este artículo trata de esclarecer, precisamente, esa segunda cuestión: ¡cuál es la relevancia ética de la edad como criterio de triaje durante la pandemia de la COVID-19?

La edad es un marcador cronológico con repercusiones médicas. A veces se tiene en cuenta en la priorización de máquinas de hemodiálisis, órganos escasos, cirugías electivas, etcétera. Para algunas voces, la edad debe considerarse como criterio relevante para racionar la ventilación mecánica intensiva (VMI) en pacientes con SARS-CoV-2 $2^{1}$ (Miller, 2020). Otros, en cambio, creen que su uso como criterio de triaje es una de las prácticas edadistas más extremas de la pandemia (Ayalon et al., 2020). Las diversas guías profesionales y protocolos éticos también han ofrecido formulaciones dispares. La Sociedad Italiana de Anestesia, Analgesia, Reanimación y Terapia Intensiva (SIAARTI) fue de las primeras en sugerir la necesidad de marcar un límite de edad para el ingreso en las unidades de cuidados intensivos (UCI) (Vergano et al., 2020). En España, la Sociedad Española de Medicina Intensiva, Crítica y Unidades Coronarias recomendaba no asignar preferentemente la VMI a pacientes mayores de 80 años y asignarla a los pacientes de entre 70 y 80 , siempre y cuando no tuviesen comorbilidades moderadas o graves (SEMICYUC, 2020). El Hospital Vall d'Hebron, igualmente, sugería que los mayores de 80 años no recibiesen VMI y que para su asignación entre los de 70 y 80 se tuviese en

1. Acrónimo en inglés de la neumonía Severe Acute Respiratory Syndrome Coronavirus 2. 
cuenta el nivel clínico de fragilidad desde la escala de Rockwood (Vall d'Hebron, 2020). El informe del Ministerio de Sanidad sobre los aspectos éticos en situación de pandemia, por su parte, señala que utilizar la edad como razón única de exclusión es discriminatorio, ya que atenta contra los derechos básicos -en especial contra el artículo 14 de la Constitución- (Ministerio de Sanidad, 2020).

En este artículo se defenderá que la edad no debe ser un criterio categórico de exclusión, aunque sí que se debe tener en cuenta debido a su presencia implícita en otros principios de triaje. Para ello se comenzará clarificando el contexto de debate. Posteriormente, se analizará cómo la equidad requiere en cierta medida dar más oportunidades a aquellos pacientes que han cumplido menos etapas vitales. A continuación, se mostrará la función de la edad a la hora de maximizar eficientemente los beneficios de los recursos escasos en situación pandémica. Finalmente, se concluirá en favor de sistemas multiprincipios de triaje, entre los que la edad está incluida como criterio no limitante.

\section{Clarificando el contexto de debate: de los criterios de triaje al triaje de criterios}

La asignación de recursos médicos escasos es casi siempre una fuente de decisiones difíciles. Algunas veces, incluso, nos precipita ante dilemas trágicos, esto es, ante decisiones de vida o muerte. El triaje durante la pandemia de la COVID-19 ha generado un desafío mayúsculo en el que varios dilemas trágicos se han vuelto desafortunadamente rutinarios. El triaje es un proceso de clasificación de pacientes según la necesidad terapéutica y el orden de gravedad. Proviene de la medicina militar y se realiza de manera ordinaria en los servicios de urgencias y emergencias (Gómez Jiménez, 2003). En tanto el triaje implica la distribución de recursos, se puede hablar de priorización o de racionamiento (cf. Hortal et al., 2020; Melguizo et al., 2020). Priorizar es establecer el orden de acceso a un recurso. Racionar, por otro lado, es elegir quién obtendrá el recurso de entre los pacientes que se pueden beneficiar de él. Estos tres términos se utilizan a menudo indistintamente en la práctica cotidiana.

Estos procesos nos sitúan en los dominios pantanosos de la justicia distributiva, es decir, en el debate sobre cómo distribuir aquellos bienes que se valoran socialmente. ¿Pero de qué acceso a recursos estamos hablando? Hay al menos tres acciones de triaje que han creado sendos conflictos morales:

1. La admisión o la exclusión de pacientes de residencias en los hospitales o en las UCI.

2. La admisión o la exclusión en las UCI.

3. La asignación o la no asignación - ya sea mediante la instauración o la retirada - de la VMI. 
Como cada uno de estos tres procedimientos merece atención pormenorizada, en el presente artículo se acotará el análisis al racionamiento de la VMI. No obstante, muchas de las siguientes consideraciones pueden trasladarse a los otros dos casos, debido a que la edad de los pacientes ha sido importante en esos mismos debates.

La VMI es el tratamiento crítico de soporte vital más importante para los pacientes graves con SARS-CoV-2 (Yang et al., 2020: 480). Es una terapia invasiva en la que se intuba al paciente (normalmente con síndrome de distrés respiratorio agudo o neumonía intersticial caracterizada por hipoxemia severa) para que el respirador artificial asista mecánicamente la ventilación pulmonar. En pocas palabras, la VMI soporta el funcionamiento del sistema respiratorio, sin el cual es imposible continuar viviendo. Cabe mencionar, de hecho, que la asignación de los ventiladores es una cuestión de vida o muerte inminente, debido a que al paciente a quien se le deniega la VMI puede morir incluso en cuestión de minutos (Truog et al., 2020). Dicho esto, ¿cuáles son los principios éticos que se deben tener en cuenta para la asignación de la VMI?, ¿en qué criterios se desglosan estos principios? y ¿qué lugar ocupa en ellos la edad?

El doble eje en el que pivotan los criterios de triaje se mueve primordialmente entre dos principios: la equidad y la eficiencia (Graf et al., 2020; Hortal et al., 2020; López de la Vieja y Rodríguez-Arias, 2020; Melguizo et al., 2020; Wilkinson, 2020). La equidad consiste, en cierto modo, en la justicia entendida como igualdad de oportunidades. La eficiencia, por otro lado, trata de maximizar el beneficio (fin) de los recursos disponibles (medios). Estos dos principios consideran la edad, como veremos, con una relevancia éticamente diferente. Asimismo, estos principios engloban criterios de distribución diversos. La equidad da cabida a criterios que pueden priorizar a personas según la etapa de la vida en la que se encuentren, a embarazadas, a los más jóvenes, a niños y niñas o al paciente más grave. Si se considera la equidad como el trato estrictamente igualitario, este principio puede incluir criterios como la lotería o el orden de llegada (first-come, firstserved). La eficiencia, por otro lado, trata de maximizar principalmente los siguientes objetivos: salvar vidas (supervivencia a corto plazo y conseguir el alta) y salvar años de vida (supervivencia a largo plazo). La eficiencia también intenta minimizar el tiempo de uso de un recurso - siempre que se consiga el mismo resultado o uno mejor con menos tiempo de uso-. Puede tener en cuenta, igualmente, el valor instrumental que puede aportar a corto plazo la priorización de ciertos trabajadores esenciales de primera línea durante la pandemia - la utilidad social estrecha- o, de manera más controvertida, la de personas con valor a largo plazo para la sociedad en general — la utilidad social extendida- (Jecker et al., 2020).

Ahora bien, no todo criterio es igual de importante a nivel ético. Curiosamente, los expertos en triaje también recomiendan la priorización de criterios ${ }^{2}$. Por ejemplo, hay bastante insistencia en no utilizar el orden de llegada (Persad

2. Esto es extensible al debate sobre el racionamiento de las vacunas. Véase Cruz et al. (2020). 
et al., 2009; White et al., 2009; Emanuel et al., 2020; Hortal et al., 2020). Otros criterios, asimismo, no se consideran de primer orden, pero sí susceptibles de tenerse en cuenta cuando el puntaje de triaje de dos pacientes es similar. Un criterio de desempate recurrente es el sorteo o la lotería (White et al., 2009; Emanuel et al., 2020; Matheny Antommaria et al., 2020; Savulescu et al., 2020). La edad también se ha sugerido como primer mecanismo de desempate (Daugherty Biddison et al., 2019). Dicho esto, en lo que sigue, este artículo se ceñirá a tres de los criterios más importantes, los cuales guardan relación con la relevancia de la edad: los ciclos de vida, la búsqueda de beneficio a corto plazo y la búsqueda de beneficio a largo plazo.

\section{Equidad, ciclos de vida y justicia intergeneracional}

La equidad es un término estrictamente ético-político - a diferencia de la noción de eficiencia, en la que el beneficio se valora desde la imbricación de razones éticas, clínicas y económicas-. La equidad trata de distribuir las oportunidades de manera justa y se puede concebir de dos formas sustancialmente diferentes: o bien la equidad consiste en tratar a todos por igual, o bien permite el trato desigual (acción o discriminación positiva) para garantizar la igualdad de oportunidades. Esta segunda visión es la más destacable respecto a la consideración de la edad en los debates de racionamiento. Por eso se debe atender al argumento ético que respalda esa posición — los fair innings- y su correspondiente traducción a criterios de triaje —el de los ciclos de vida—. Se comenzará con el argumento.

El argumento de los fair innings ${ }^{3}$ viene a mostrar que la edad tiene un carácter moralmente significativo relacionado con la justicia intergeneracional. Según John Harris (1985: cap. 5), este argumento sugiere que todos deberíamos tener el derecho a llegar al umbral (digamos unos 70 años) que permite la realización de proyectos vitales comunes y la consecución de una vida plena. A partir de dicho límite, las oportunidades que se deben garantizar para seguir viviendo decrecen, sobre todo si se compite por recursos médicos críticos con personas más jóvenes. Si bien la vida es valiosa en todas sus etapas y su final no deseado es una desgracia, la muerte de una vida joven además de un infortunio es una tragedia injusta. Este argumento, por lo tanto, da razones morales para priorizar tratamientos a las personas que hayan tenido menos oportunidades de completar las principales etapas vitales.

Este argumento tiene su correspondiente versión práctica en el criterio de los ciclos de vida ${ }^{4}$. Básicamente, este reclama que las personas deberían tener la oportunidad de pasar por diferentes etapas de la vida (Persad et al., 2009). La asignación de recursos en situación pandémica debe tener en cuenta en qué

3. Los innings son segmentos de juego en deportes como el béisbol o el críquet, en los que los equipos van cambiando el turno de bateo para atacar o defender. La expresión fair innings forma pare del folklore popular británico, aunque recoge una idea también presente en la teología cristiana (Evans, 1997; Williams, 1997).

4. Una versión emparentada es el criterio de priorizar a los más jóvenes. 
ciclo se encuentra el paciente destinatario, dando mayor prioridad a aquellos que han cumplido menos etapas vitales (Emanuel y Wertheimer, 2006). Esto se puede traducir en un puntaje de triaje diferenciado según grupos de edad $\left(0-49^{5}, 50-69,70-84,>85\right)$, en el que se premia a quienes han vivido menos (Daugherty Biddison et al., 2019).

Este criterio tiene al menos dos ventajas. Primero, encaja de manera adecuada con intuiciones popularmente extendidas ${ }^{6}$. Gran cantidad de estudios empíricos muestran que hay una aceptación social mayoritaria sobre la permisibilidad de priorizar ciertos recursos médicos entre los más jóvenes en detrimento de los más ancianos (cf. Bognar, 2015: 252n3). Segundo, tiene una dimensión inherentemente igualitarista (White et al., 2009: 135). A diferencia del género o la raza, el envejecimiento es un proceso biológico insoslayable que nos iguala como seres humanos. Además, este criterio exalta el valor de vivir en cada estadio vital y demanda que hay que brindar oportunidades para distribuir equitativamente el mayor número de ciclos.

Esta posición, no obstante, tiene también dos desventajas. Por un lado, la delimitación del umbral es arbitraria. La dificultad de trazar una línea entre diferentes grupos de edad se hace palmaria, por ejemplo, cuando los umbrales recomendados podrían hacer priorizar los recursos en una persona de 49 años en vez de en una de 50, aun cuando el resto de los requisitos sean iguales (Daugherty Biddison et al., 2019). De hecho, esto es algo que ya ocurre de manera sesgada en la distribución médica de algunos tratamientos. El left digitbias es la tendencia a categorizar variables continuas en función del número que está más a la izquierda (Olenski et al., 2020). Un cambio de dígito puede hacer que algo que cuesta 1 euro parezca notoriamente más caro que si cuesta 0,99 euros, cuando el incremento es en realidad nimio. Así, por ejemplo, Olenski et al. (2020) mostraron que los pacientes admitidos dos semanas antes de cumplir los 80 años tenían más probabilidades de ser sometidos a una revascularización quirúrgica que los que tenían 80 años y dos semanas, aunque no hubiera diferencias clínicas relevantes de por medio. Este sesgo puede haber afectado a la concesión de recursos durante la pandemia (Nickel et al., 2020), volviendo todavía más problemáticos aquellos filtros (fijados en cambios de décadas) que se aplicaron de manera excluyente.

Por otro lado, ligar el cumplimiento de etapas a la satisfacción vital es discutible, especialmente si se derivan exigencias de justicia. Superar la cuota

5. Este lapso abarca etapas con diferencias notables. Para algunos habría razones morales para priorizar a adultos de 20 años en vez de infantes, debido a que la muerte de los primeros supondría una frustración de mayores planes y proyecciones vitales (Emanuel y Wertheimer, 2006: 854-855). Sin embargo, en lo que respecta al triaje de la VMI, los pacientes pediátricos no entrarían en este debate, ya que utilizan otros ventiladores específicos y un personal asistencial diferente (White et al., 2009: 135).

6. El sondeo moral más importante de este siglo —el Moral machine experiment sobre situaciones dilemáticas a las que se enfrentan los coches autónomos - ha mostrado recientemente una fuerte preferencia global por salvar las vidas de los más jóvenes (Awad et al., 2018). Sin embargo, también ha mostrado que la importancia del criterio de la edad varía según regiones, ya que el valor de la senectud puede cambiar de una cultura a otra. 
de ciclos de vida justos establece una relación débil con la satisfacción personal, mayormente porque esta tiene que ver más con las vivencias particulares de cada cual que con la etapa en la que uno se encuentre. Algunas personas llegan a sus fair innings habiendo tenido una vida plena, otras teniendo una existencia aciaga. Para algunas, la jubilación puede ser una etapa dorada; para otras, una época de precariedad. Así, lo que importa no es la cantidad de años vividos, sino la calidad con los que se ha vivido esos años (Williams, 1997: 822). En este sentido, tratar de hacer justicia a todas las contingencias personales está condenado al fracaso de antemano. Este contraargumento sugiere, entonces, que el atractivo intuitivo de los fair inning puede frustrarse en algunos casos particulares.

En resumen, el criterio de los ciclos de vida se apoya en la equidad para dar cierta prioridad a los más jóvenes. Vistas las deficiencias que se le pueden presentar, esta prioridad no debe ser nunca absoluta, sino tan solo débil, y cojea sin el apoyo justificatorio de otros criterios. La equidad, asimismo, es una brújula normativa que exige evitar discriminaciones sistemáticas y favorecer, en algunas situaciones, a colectivos vulnerables (Hortal et al., 2020). Si bien hay razones de justicia para dar oportunidades a los que han vivido menos, esto no puede hacerse a costa de la exclusión categórica de los que se encuentran en los últimos estadios vitales.

\section{Eficiencia en la escasez: maximizando el beneficio de los recursos disponibles}

La edad no es solo importante porque un paciente de edad avanzada ya puede haber cumplido los ciclos de vida principales, sino también porque afecta a las capacidades de un individuo para beneficiarse de un tratamiento (Williams, 1997). Esta segunda consideración es todavía más importante cuando la demanda desborda la limitada oferta de servicios, como ha ocurrido con la VMI (Ranney et al., 2020; Wells et al., 2020). En situación de abundancia de medios, el principio de equidad obliga a dar oportunidades a toda persona que se pueda beneficiar de los recursos médicos. Sin embargo, la carencia extrema de la pandemia impele a subordinar por razones éticas la equidad a la eficiencia (Graf et al., 2020; Savulescu et al., 2020; Wilkinson, 2020), es decir, a distribuir los recursos escasos buscando el máximo beneficio total.

La eficiencia está ligada a cuestiones técnico-clínicas y de economía de la salud, pero también a razones éticas. De hecho, los debates de triaje son de raigambre utilitarista (Jonsen, 1986; Solnica et al., 2020). El utilitarismo entiende la eficiencia del triaje como utilidad: maximizar el beneficio total agregado de las decisiones de priorización (cf. Savulescu et al., 2020). Este planteamiento puede suscitar, por lo menos, dos sospechas. Primero, el mayor bien para el mayor número puede discriminar a colectivos vulnerables que reciben menor prioridad debido a que generan menor beneficio total. Segundo, la forma de medir el beneficio es un asunto cuanto menos controvertido. Estas dos cuestiones repercuten en la relevancia de la edad en el debate de triaje. 
El beneficio se mide principalmente a corto y a largo plazo (McGuire et al., 2020). De estos dos polos derivan criterios de triaje de primer orden: salvar vidas y salvar años de vida (Emanuel et al., 2020). Para salvar vidas se intenta establecer la probabilidad de supervivencia a corto plazo, esto es, un juicio pronóstico sobre el estado del paciente y su potencial para sobrevivir al tratamiento y conseguir el alta posteriormente. Para salvar años de vida, por otro lado, se trata de maximizar la supervivencia a largo plazo tras el alta hospitalaria. Esta segunda expectativa puede tener en cuenta cálculos de coste-efectividad como los años de vida ajustados por calidad (AVAC) o los años de vida ajustado por discapacidad (AVAD). En este sentido, buscar el beneficio a largo plazo trata de rescatar algo moralmente muy valioso: ganar cuantos más años de vida con la mejor calidad posible. No obstante, en la comparación interpersonal del triaje esto pone en desventaja a los pacientes de mayor edad, a personas con diversidad funcional e incluso a personas de niveles socioeconómicos bajos, que - debido a los determinantes sociales de la salud - tienen más comorbilidades y menor esperanza de vida (McGuire et al., 2020; Melguizo et al., 2020).

Salvar vidas es otro objetivo éticamente deseable que se debe maximizar. Saber si alguien tiene posibilidades de sobrevivir y de recuperarse a corto plazo es algo que requiere pericia clínica y conocimiento experto. Por un lado, uno de los sistemas de puntaje que más se utiliza como juicio pronóstico a corto plazo en las UCI es el Sequential Organ Failure Assesment (SOFA). El SOFA ofrece datos dinámicos objetivos (basados en métricas fisiológicas y bioquímicas) con bastante validez predictiva sobre el riesgo de mortalidad de los pacientes adultos ingresados - en niños se utiliza el Pediatric Logistic Organ Dysfunction (PELOD) - . A pesar de que el SOFA evita que se usen factores únicos (como la edad o la discapacidad) para excluir a los pacientes de los recursos, sus pronósticos deben combinarse con el juicio clínico experto para excluir arbitrariedades éticamente indeseables (McGuire et al., 2020).

Por otro lado, los profesionales sanitarios utilizan heurísticas en sus decisiones rutinarias de adjudicación de recursos (McDonald, 1996). Algunas heurísticas funcionan como atajos cognitivos para abreviar el razonamiento y ahorrar tiempo (Olenski et al., 2020). Estas estrategias son «frugales», debido a que se centran en una parte de la información disponible (p. ej.: en variables o predictores simples como la edad, la comorbilidad o la fragilidad) para agilizar la toma de decisiones (Wegwarth et al., 2009). La experticia médica y el «ojo clínico» se basan en cierta medida en este tipo de heurísticas ${ }^{7}$. ¿Qué papel puede jugar la edad al respecto? La edad es un buen predictor (no un mecanismo determinante) del incremento de problemas relacionados con la salud: indica una disminución de la capacidad de recuperación, mayor necesidad de atención especializada, menor probabilidad de beneficiarse de un tratamiento, menor esperanza de vida tras el mismo, etc. (Williams, 1997: 821).

7. A pesar de lo resolutivas que son la mayoría de las heurísticas, algunas de ellas pueden estar sesgadas. El left-digit bias que hemos visto en el anterior apartado es una de dichas tendencias problemáticas. 
La edad es también un indicador a tener en cuenta en la asignación de la VMI a los pacientes con SARS-CoV-2. Si bien los cuidados intensivos suponen un desgaste para cualquier persona, la VMI exige resistencia por parte de los enfermos más graves. Este tratamiento invasivo requiere de intubación endotraqueal mediante un laringoscopio y anestesia general, pudiendo necesitarse varias semanas ${ }^{8}$ de soporte en estado inconsciente (incluso en posición decúbito prono) y sin poder alimentarse oralmente, lo que provoca una gran pérdida de masa corporal y un deterioro físico notable. Las personas mayores más frágiles son las que menos se benefician de estos tratamientos, con una tasa de supervivencia baja a partir de los 70 años y una gran mortalidad con más de 80 (Richardson et al., 2020; Yang et al., 2020; Wu y McGoogan, 2020) ${ }^{9}$. Obviamente, la capacidad de beneficiarse menos no depende solo de la edad, sino también del estado de salud en general del paciente, comorbilidades y fragilidad clínica incluidas.

Nickel et al. (2020) sugieren, de hecho, que el estado de fragilidad es un mejor indicador que la edad en medicina geriátrica respecto a los efectos de la COVID-19. Recomiendan el uso de la escala de fragilidad clínica (Clinical Frailty Scale), un predictor de mortalidad dentro del hospital independiente de la edad y el género, el cual puede ser una guía menos sesgada que la edad para la asignación de recursos en las UCI. Igualmente, la considerable variación interpersonal de salud en el envejecimiento demanda un juicio individualizado de riesgos y beneficios (Evans, 1997: 314). No todos los ancianos son igual de vulnerables al SARS-CoV-2. En China se reportaron entre febrero y marzo altas de tres pacientes de 98 años y de dos centenarios (de $101 \mathrm{y}$ 103 años) - algunos de los cuales requirieron cuidados intensivos, pero ninguno VMI- (Huang et al., 2020). Estos casos son recordatorios de que hay mayores de 80 años con «salud de hierro» que tienen probabilidad de beneficiarse sobreviviendo a corto plazo y que pueden llegar a tener una esperanza de vida apreciable a largo plazo, aunque sean una escasa minoría.

Por lo tanto, tratar de maximizar la eficiencia no puede servir de pretexto para excluir categóricamente a individuos por el hecho arbitrario de pertenecer a un colectivo de edad en el que a priori se genera menor beneficio. La correlación entre mayor edad, comorbilidad y fragilidad no debe usarse como un predictor contundente, ya que se debe evitar a toda costa penalizar precisamente a los ancianos sanos que sí que se pueden beneficiar a corto y largo plazo (White et al., 2009: 136). En este sentido, los filtros de exclusión por edad

8. El tiempo de consumo de un recurso también se debe tener en cuenta a la hora de maximizar la utilidad (Savulescu et al., 2020). Si se pronostica que el paciente A requiere el ventilador una semana y el paciente B lo requiere durante dos semanas, podemos tener una razón añadida para priorizar a $\mathrm{A}$. Tras liberarse el ventilador, este podría ser asignado al paciente $\mathrm{C}$, quien también tiene un pronóstico de uso de una semana. Así, esta decisión de triaje podría salvar a A y $\mathrm{C}$ en el mismo plazo que si solo se intentara salvar a B.

9. Una cuestión que en este artículo no hay espacio para abordar es si aplicar la VMI a personas con probabilidades nimias de supervivencia supondría un encarnizamiento terapéutico y una mala muerte. 
fueron una recomendación éticamente desacertada. Darle tal carácter distintivo a la edad no se justifica desde un prisma ético. La urgencia temporal de las decisiones en los momentos de colapso tampoco es excusa para denegar $e x$ ante recursos vitales a personas que pueden beneficiarse de ellos. Los criterios éticos principales para denegar el acceso al recurso son que haya pacientes con mayor probabilidad de supervivencia a corto plazo, mejor expectativa a largo plazo y que hayan cumplido menos ciclos vitales. En caso de que haya «empate» en el puntaje de triaje tras considerar esos tres factores se puede recurrir al sorteo como criterio final.

\section{Conclusión: la edad como categoría no excluyente}

La edad por sí sola no debe ser un criterio de triaje protagonista, puesto que solo es relevante en cuanto se trata de una variable secundaria a tener en cuenta dentro de los principios de equidad y beneficio. Las categorías y los filtros de exclusión son discriminaciones a ciegas que se deben evitar a toda costa (Auriemma et al., 2020), incluido el caso de la edad (Donnelly, 2020). Igualmente, para la distribución de recursos escasos no valen los principios únicos (Persad et al., 2009). Existe un consenso amplio en que el triaje requiere de la aplicación concertada de múltiples criterios (Persad et al., 2009; White et al., 2009; Daugherty Biddison et al., 2018 y 2019; Emanuel et al., 2020; Hortal et al., 2020; Melguizo et al., 2020). Además, ya hay aportaciones empíricas que muestran que hay una mayor aceptación pública del uso compuesto de criterios en detrimento del uso de categorías simples de priorización (Rueda et al., 2020). En definitiva, los juicios de triaje deben hacerse al nivel individualizado del paciente, sin aplicar exclusiones sistemáticas y promoviendo los valores codificados en los diversos criterios éticos de racionamiento.

Finalmente, la narrativa social pospandémica debe hilvanar una visión conciliadora, debilitando las manifestaciones edadistas (y gerontofóbicas) para fortalecer la solidaridad intergeneracional (Ayalon et al., 2020). Si algunas muestras de desprotección a los más vulnerables y a nuestros ancianos menguaron la confianza pública en el racionamiento sanitario, hay que dar pasos para reconstruir esos puentes caídos. La edad no debió sugerirse como última ratio para las decisiones de triaje. Se deben reconocer los desaciertos y hay que anticiparse colectivamente para que no se repitan más en el futuro.

\section{Referencias bibliográficas}

Auriemma, C.L.; Molinero, A.M.; Houtrow, A.J. y Persad, G. (2020). "Eliminating categorical exclusion criteria in crisis standards of care frameworks». The American Journal of Bioethics, 20 (7), 28-36. $<$ https://doi.org/10.1080/15265161.2020.1764141>

Awad, E.; Dsouza, S.; Kim, R. et al. (2018). «The Moral Machine experiment». Nature, 563, 59-64. $<$ https://doi.org/10.1038/s41586-018-0637-6> 
Ayalon, L.; Chasteen, A.; Diehl, M.; Levy, B.R.; Neupert, S.D.; Rothermund, K.; Tesch-Römer, C. y Wahl, H.-W. (2020). «Aging in Times of the COVID-19 Pandemic: Avoiding Ageism and Fostering Intergenerational Solidarity». The Journals of Gerontology: Series B, gbaa051. <https://doi.org/10.1093/geronb/gbaa051>

Bognar, G. (2015). «Fair innings». Bioethics, 29 (4), 251-261. $<$ https://doi.org/10.1111/bioe.12101>

Cruz, M.; Hortal, J. y Padilla, J. (2020). "“Vísteme despacio que tengo prisa”: Un análisis ético de la vacuna del COVID-19: fabricación, distribución y reticencia». Enrahonar, 65, 57-73.

Daugherty Biddison, E.L.D.; Faden, R.; Gwon, H.S. et al. (2019). «Too many patients...: A framework to guide statewide allocation of scarce mechanical ventilation during disasters». CHEST, 155 (4), 848-854. <https://doi.org/10.1016/j.chest.2018.09.025>

Daugherty Biddison, E.L.D.; Gwon, H.S.; Schoch-Spana, M. et al. (2018). «Scarce Resource Allocation During Disasters: A Mixed-Method Community Engagement Study». CHEST, 153 (1), 187-195. $<$ https://doi.org/10.1016/j.chest.2017.08.001>

Donnelly, S. (2020). «The Elderly \& COVID-19: Cocooning or Culling: - the choice is ours». QJM: An International Journal of Medicine, 113 (7), 453-454. <https://doi.org/10.1093/qjmed/hcaa145>

Emanuel, E.J.; Persad, G.; Upshur, R. et al. (2020). «Fair allocation of scarce medical resources in the time of COVID-19». The New England Journal of Medicine, 382, 2049-2055.

Emanuel, E.J. y Wertheimer, A. (2006). «Who should get influenza vaccine when not all can?». Science, 312 (5775), 854-855. <https://doi.org/10.1126/science.1125347>

Evans, J.G. (1997). «The rationing debate: Rationing health care by age: The case against». British Medical Journal, 314, 822-825.

Gómez Jiménez, J. (2003). «Clasificación de pacientes en los servicios de urgencias y emergencias: Hacia un modelo de triaje estructurado de urgencias y emergencias». Emergencias, 15, 165-174.

Graf, W.D.; Epstein, L.G. y Pearl, P.L. (2020). «Practical Bioethics during the Exceptional Circumstances of a Pandemic». Pediatric Neurology, 108, 3-4.

<https://doi.org/10.1016/j.pediatrneurol.2020.04.011>

Harris, J. (1985). The value of life: An introduction to medical ethics. Londres: Routledge, 2001.

Hortal, J.; Padilla, J.; Melguizo, M. et al. (2020). «La eficiencia no basta: Análisis éticos y recomendaciones para la distribución de recursos escasos en situación de pandemia». Gazeta sanitaria, en prensa.

Huang, Y.M.; Hong, X.Z.; Shen, J.; Huang, Y. y Zhao, H.L. (2020). "China's oldest coronavirus survivors". Journal of the American Geriatrics Society, 68 (5), 940-942. 
Jecker, N.S.; Whigtman, A.G. y Diekeman, D.S. (2020). «Prioritizing frontline workers during the COVID-19 pandemic». The American Journal of Bioethics, 20 (7), 128-132. <https://doi.org/10.1080/15265161.2020.1764140>

Jonsen, A.R. (1986). «Bentham in a box: Technology assessment and health care allocation». Law, Medicine \& Healthcare, 14 (3-4), 172-174.

López de LA Vieja, M.T. y Rodríguez-Arias, D. (2020). «Emergencia sanitaria: Dos marcos de deliberación». Enrahonar, 65, 37-46.

Matheny Antommaria, A.H.; Gibb, T.S.; McGuire, A.L. et al. (2020). «Ventilators triage policies during COVID-19 pandemic at U.S. hospitals associated with members of the Association of Bioethics Program Directors». Annals of Internal Medicine, 173 (3), 188-194. <https://doi.org/10.7326/M20-1738>

McDonald, C.J. (1996). "Medical heuristics: The silent adjudicators of clinical practice». Annals of Internal Medicine, 124 (1 Pt 1), 56-62.

McGuire, A.; Aulisio, M.P.; Davis, F.D.; Erwin, C.; Harter, T.D.; Jagsi, R.; Kuitzman, R.; Macauley, R.; Racine, E.; Wolf, S.M. et al. (2020). «Ethical challenges arising in the COVID-19 pandemic: An overview from the Association of Bioethics Program Directors (ABPD) task force». The American Journal of Bioethics, 20 (7), 15-27. <https://doi.org/10.1080/15265161.2020.1764138>

Melguizo, M.; Hortal, J. y Padilla, J. (2020). «Medicina basada en las "existencias" o soporte ético para las decisiones difíciles». Actualización en Medicina de Familia. Recuperado de <https://amf-semfyc.com/web/article_ ver.php?id=2685>.

MilleR, F.G. (2020). «Why I support age-related rationing of ventilators for COVID-19 patients». Hasting Bioethics Forum (9 de abril). Recuperado el 15 de junio de 2020, de <https://www.thehastingscenter.org/why-isupport-age-related-rationing-of-ventilators-for-covid-19-patients/>.

Ministerio de Sanidad (2020). Informe del Ministerio de Sanidad sobre los aspectos éticos en situación de pandemia: El SARS-CoV-2 (3 de abril). Recuperado el 15 de junio de 2020, de <https://www.mscbs.gob.es/profesiona les/saludPublica/ccayes/alertasActual/nCov-China/documentos/Aspectos Eticos_en_situaciones_de_pandemia.pdf>.

Nickel, C.H.; Ruegg, M.; Pargger, H. y Bingisser, R. (2020). «Age, comorbidity, frailty status: Effects on disposition and resource allocation during the COVID-19 pandemic». Swiss Medical Weekly, 150, w20269. $<$ https://doi.org/10.4414/smw.2020.20269>

Olenski, A.; Zimerman, A.; Coussens, S. y Jena, A.B. (2020). «Behavioural heuristics in coronary-artery bypass graft surgery». The New England Journal of Medicine, 382 (8), 778-779.

Persad, G.; Wertheimer, A. y Emanuel, E.K. (2009). «Principles for allocation of scarce medical interventions». The Lancet, 373, 423-431.

Ranney, M.L.; Griffeth, V. y JhA, A.K. (2020). "Critical supply shortages - The need for ventilators and personal protective equipment during the 
COVID-19 pandemic». The New England Journal of Medicine, 382, e41. <https://doi.org/10.1056/NEJMp2006141>

Richardson, S.; Hirsch, J.S.; Narasimhan, M. et al. (2020). "Presenting Characteristics, Comorbidities, and Outcomes Among 5700 Patients Hospitalized With COVID-19 in the New York City Area». Journal of the American Medical Association, 323 (20), 2052-2059.

Rueda, J.; Hannikainen, I.; Hortal, J. y Rodríguez-Arias, D. (2020). «Examining public trust in categorical versus comprehensive triage criteria». The American Journal of Bioethics, 20 (7), 106-109. <https://doi.org/10.1080/15265161.2020.1779867>

Savulescu, J.; Persson, I. y Wilkinson, D. (2020). «Utilitarianism and the pandemic». Bioethics, 34 (6), 620-632. $<$ https://doi.org/10.1111/bioe.12771>

SEMICYUC (2020). Recomendaciones éticas para la toma de decisiones en la situación excepcional de crisis por pandemia COVID-19 en las unidades de cuidados intensivos. Recuperado el 15 de junio de 2020, de <https://semicyuc.org/ wp-content/uploads/2020/03/\%C3\%89tica_SEMICYUC-COVID-19.pdfs.

Solnica A.; Barski, L. y Jotkowitz, A. (2020). «Allocation of scarce resources during the COVID-19 pandemic: A Jewish ethical perspective». Journal of Medical Ethics, 46 (7), 444-446.

<http://dx.doi.org/10.1136/medethics-2020-106242>

Truog, R.D.; Mitchell, C.R.N. y Daley, G.Q.M.D. (2020). «The toughest triage - allocating ventilators in a pandemic». The New England Journal of Medicine, 382, 1973-1975.

Vall d'Hebron (2020). Recomanacions per suport a les decisions d'adequació de l'esforç terapèutic (AET) per pacients amb sospita d'infecció per COVID-19 insuficiència respiratòria aguda (IRA) hipoxèmica. Grup de treball de Ventilació, Cap Àmbit Emergències, Direcció Mèdica i Direcció Infermera de SEM (25 de marzo). Recuperado el 15 de junio de 2020, de <https://coronavirus.coib. cat/wp-content/uploads/2020/03/recomanacions-suport-decisions-aet.pdfs.

Vergano, M.; Bertolini, G.; Giannini, A.; Gristina, G.; Livigni, S.; Mistraletti, G. y Petrini, F. (2020). "Clinical ethics recommendations for the allocation of intensive care treatments, in exceptional, resource-limited circumstances». SIAARTI (16 de marzo). Recuperado el 15 de junio de 2020, de <http://www.siaarti.it/SiteAssets/News/COVID19\%20-\%20 documenti\%20SIAARTI/SIAARTI\%20-\%20Covid-19\%20-\%20Clini cal\%20Ethics\%20Reccomendations.pdf>.

Wegwarth, O.; Gaissmaier, W. y Gigerenzer, G. (2009). «Smart strategies for doctors and doctors-in-training: Heuristics in medicine». Medical Education, 43 (8), 721-728. <https://doi.org/10.1111/j.1365-2923.2009.03359.x>

Wells, C.R.; FitzPatrick, M.C.; Sah, P. et al. (2020). «Protecting the demand for ventilators at the peak of the COVID-19 outbreak in the USA». The Lancet: Infectious Diseases. <https://doi.org/10.1016/S1473-3099(20)30315-7> 
White, D.B.; Katz, M.H.; Luce, J.M. y Lo, B. (2009). «Who should receive life support during a public health emergency?: Using ethical principles to improve allocation decisions». Annals of Internal Medicine, 150 (2), 132-139.

WiLKINSON, D. (2020). «ICU triage in an impending crisis: Uncertainty, preemption and preparation». Journal of Medical Ethics, 46 (5), 287-288. <http://dx.doi.org/10.1136/medethics-2020-106226>

Williams, A. (1997). "The rationing debate: Rationing health care by age: The case for». British Medical Journal, 314, 820-822.

Wu, Z. y McGoogan, J.M. (2020). "Characteristics of and important lessons from the coronavirus disease 2019 (COVID-19) outbreak in China: Summary of a report of 72314 cases from the Chinese Center for Disease Control and Prevention». Journal of the American Medical Association, 323 (13), 1239-1242.

YANG, X.; YU, Y.; XU, J. et al. (2020). «Clinical course and outcomes of critically ill patients with SARS-CoV-2 pneumonia in Wuhan, China: A singlecentered, retrospective, observational study». The Lancet. Respiratory Medicine, 8 (5), 475-481.

Jon Rueda Etxebarria es investigador predoctoral en la Unidad Científica de Excelencia FiloLab de la Universidad de Granada, gracias a una beca "La Caixa" INPhINIT Retaining. Es graduado en Filosofía por la Universidad de Salamanca y tiene un máster en Ética y Democracia por la Universitat de València. Ha disfrutado de un contrato de 18 meses como Joven Personal Investigador (Garantía Juvenil) en el proyecto BIOethAI+ en la Universidad de Granada. Sus líneas de investigación principales son la bioética práctica y la mejora humana.

Jon Rueda Etxebarria is a PhD candidate at the FiloLab Scientific Unit of Excellence of the University of Granada thanks to a La Caixa INPhINIT Retaining Fellowship. He holds a Bachelor of Philosophy from the University of Salamanca and a Master of Ethics and Democracy from the University of Valencia. He has enjoyed an 18-month contract as a Research Assistant (Youth Guarantee) in the BIOethAI + project at the University of Granada. His main lines of research are practical bioethics and human enhancement. 